\title{
Bifurcation analysis in a vortex flow generated by an oscillatory magnetic obstacle
}

\author{
Beltrán, A.; Ramos, E.; Cuevas, S.; Brøns, Morten
}

Published in:

Physical Review E

Link to article, DOI:

10.1103/PhysRevE.81.036309

Publication date:

2010

Document Version

Publisher's PDF, also known as Version of record

Link back to DTU Orbit

Citation (APA):

Beltrán, A., Ramos, E., Cuevas, S., \& Brøns, M. (2010). Bifurcation analysis in a vortex flow generated by an oscillatory magnetic obstacle. Physical Review E, 81(3), 036309. https://doi.org/10.1103/PhysRevE.81.036309

\section{General rights}

Copyright and moral rights for the publications made accessible in the public portal are retained by the authors and/or other copyright owners and it is a condition of accessing publications that users recognise and abide by the legal requirements associated with these rights.

- Users may download and print one copy of any publication from the public portal for the purpose of private study or research.

- You may not further distribute the material or use it for any profit-making activity or commercial gain

- You may freely distribute the URL identifying the publication in the public portal 


\title{
Bifurcation analysis in a vortex flow generated by an oscillatory magnetic obstacle
}

\author{
Alberto Beltrán, ${ }^{1, *}$ Eduardo Ramos, ${ }^{1, \dagger}$ Sergio Cuevas, ${ }^{1, \ddagger}$ and Morten Brøns ${ }^{2, \S}$ \\ ${ }^{1}$ Centro de Investigación en Energía, Universidad Nacional Autónoma de México, AP 34, Temixco, Morelos 62580, Mexico \\ ${ }^{2}$ Department of Mathematics, Technical University of Denmark, Building 303S, DK-2800 Kongens Lyngby, Denmark
}

(Received 20 August 2009; published 9 March 2010)

\begin{abstract}
A numerical simulation and a theoretical model of the two-dimensional flow produced by the harmonic oscillation of a localized magnetic field (magnetic obstacle) in a quiescent viscous, electrically conducting fluid are presented. Nonuniform Lorentz forces produced by induced currents interacting with the oscillating magnetic field create periodic laminar flow patterns that can be characterized by three parameters: the oscillation Reynolds number, $\operatorname{Re}_{\omega}$, the Hartmann number, $\mathrm{Ha}$, and the dimensionless amplitude of the magnetic obstacle oscillation, $D$. The analysis is restricted to oscillations of small amplitude and $\mathrm{Ha}=100$. The resulting flow patterns are described and interpreted in terms of position and evolution of the critical points of the instantaneous streamlines. It is found that in most of the cycle, the flow is dominated by a pair of counter rotating vortices that switch their direction of rotation twice per cycle. The transformation of the flow field present in the first part of the cycle into the pattern displayed in the second half occurs via the generation of hyperbolic and elliptic critical points. The numerical solution of the flow indicates that for low frequencies (v.e. $\operatorname{Re}_{\omega}=1$ ), two elliptic and two hyperbolic points are generated, while for high frequencies (v.e. $\operatorname{Re}_{\omega}=100$ ), a more complex topology involving four elliptic and two hyperbolic points appear. The bifurcation map for critical points of the instantaneous streamline is obtained numerically and a theoretical model based on a local analysis that predicts most of the qualitative properties calculated numerically is proposed.
\end{abstract}

DOI: 10.1103/PhysRevE.81.036309

PACS number(s): 47.65.- d

\section{INTRODUCTION}

Flows of electrically conducting liquids in localized magnetic fields present a very rich dynamic behavior. This richness has been exploited in problems such as the mixing of a passive scalar with electromagnetic forcing $[1-3]$ or the generation of fully controllable multiscale flows in laboratory $[4,5]$. Recently, it has been shown theoretically and experimentally that a uniform flow past a strongly localized magnetic field, called a magnetic obstacle, experiences an opposition produced by nonuniform Lorentz forces that may lead to steady or time-dependent flow patterns, presenting some similarities with flows past bluff bodies [6-10]. Some topological properties of the steady wake of a magnetic obstacle have been explored [9], but a detailed analysis of the topological behavior of the streamline patterns in the timedependent regime is not completed. Although quite remarkable differences exist between flows past solid and magnetic obstacles, to consider electromagnetic analogies of physical situations where solid-fluid interactions lead to interesting flow patterns may be worth exploring. That is the case, for instance, of bluff bodies in oscillatory motion within viscous fluids [11]. The analysis of the magnetic analog, namely, the flow produced when a magnetic obstacle oscillates in a quiescent conducting fluid layer, is the aim of the present study. Some aspects of motion generated by an oscillating magnetic obstacle were briefly described in [12].

The two-dimensional flow induced by an oscillatory magnetic obstacle is analyzed using a technique based on the

\footnotetext{
*albem@cie.unam.mx

†erm@cie.unam.mx

†scg@cie.unam.mx

§m.brons@mat.dtu.dk
}

identification and evolution of the instantaneous critical points. The role of the critical points for identifying structures in fluid flows has been acknowledged at least since Legendre [13]. See also [14] for a review of early work in this field. More recently, the role of critical points in complex and turbulent flows has been a focus of attention $[4,5,15]$. We will employ an efficient technique based on streamline curvature proposed by Ouellette and Gollub [16] to locate critical points.

In the present case, the harmonic motion of the magnetic obstacle generates a time-periodic localized Lorentz force that creates a vortex dipole flow that switches the direction of rotation twice per cycle. The transition between these rotation states takes place in a rather short time interval and involves the creation of elliptic and hyperbolic critical points. The particular flow topology of this transition depends on the oscillation frequency and, as it increases, bifurcations of the streamline patterns are observed, leading to more complex flow structures. The flow dynamics is analyzed numerically in order to obtain a bifurcation map that captures the topology of the flow transitions. Using a nonlinear theory, the numerical approach is complemented with a theoretical bifurcation analysis, based on the local analysis of the streamline patterns, which provides a qualitative description of the flow.

\section{FORMULATION}

Consider a two-dimensional quiescent layer of an electrically conducting, incompressible viscous fluid in the $x-y$ plane in presence of a spatially localized magnetic field $\mathbf{B}^{0}$. The magnetic field distribution is assumed to be externally imposed by a small square magnet that oscillates harmonically with a small amplitude with respect to the quiescent 
fluid. The dipolar magnetic field, for which an analytical expression exists [17], is produced by a magnetized surface uniformly polarized in the normal direction so that its magnetic moment points in the direction normal to the fluid layer. Under these conditions, the dominant contribution of the applied field comes from the component normal to the $x-y$ plane, $B_{z}^{0}$, and is the only one considered. The oscillatory motion of the applied field induces electric currents in the fluid that interact with the imposed magnetic field and produce a periodic nonhomogeneous Lorentz force that stirs the fluid and creates vorticity. The induced electric currents generate, in turn, an induced magnetic field $\mathbf{b}$, which is assumed to be much smaller than the applied field $\mathbf{b} \ll \mathbf{B}^{0}$. This means that the magnetic Reynolds number, $\mathrm{Rm}=\mu \sigma U d$, is much less than unity, a condition that is satisfied in most laboratory and industrial flows with liquid metals, molten salts, and electrolytes. Here, $\mu$ and $\sigma$ are the magnetic permeability and the electrical conductivity of the fluid, respectively. In turn $U$ and $d$ are characteristic scales of velocity and length. Since there is no imposed flow, $U$ is taken as the viscous velocity $\nu / d$, where $\nu$ is the kinematic viscosity of the fluid and $d$ is the side length of the square magnetized plate. The dimensionless equations that govern the flow dynamics are

$$
\begin{gathered}
\frac{\partial u}{\partial x}+\frac{\partial v}{\partial y}=0, \\
\operatorname{Re}_{\omega} \frac{\partial u}{\partial t}+u \frac{\partial u}{\partial x}+v \frac{\partial u}{\partial y}=-\frac{\partial p}{\partial x}+\nabla_{\perp}^{2} u+\mathrm{Ha}^{2} j_{y} B_{z}^{0}, \\
\operatorname{Re}_{\omega} \frac{\partial v}{\partial t}+u \frac{\partial v}{\partial x}+v \frac{\partial v}{\partial y}=-\frac{\partial p}{\partial y}+\nabla_{\perp}^{2} v-\mathrm{Ha}^{2} j_{x} B_{z}^{0},
\end{gathered}
$$

where the subindex $\perp$ denotes the projection of the $\nabla$ operator on the $x-y$ plane. The velocity components, $u$ and $v$, the pressure $p$, and the induced electrical current density $j_{x}$ and $j_{y}$ are normalized respectively by $U, \rho U^{2}$, and $\sigma U B_{m}$, where $\rho$ is the mass density of the fluid. The applied field $B_{z}^{0}(x, y)$ is normalized by $B_{m}$ which is the maximum magnetic field strength of the magnetic dipole. The dimensionless coordinates $x$ and $y$ are normalized by $d$ and time $t$ is normalized by the forced frequency of oscillation $\omega$. The dimensionless parameters are the Hartmann number, $\mathrm{Ha}=B_{m} d \sqrt{\sigma / \rho \nu}$, and the oscillation Reynolds number, $\operatorname{Re}_{\omega}=\omega d^{2} / \nu$.

In addition, the induced magnetic field in the quasistatic approximation is governed by the induction equation which in the two-dimensional case, neglecting $O(\mathrm{Rm})$ terms, reduces to

$$
\nabla_{\perp}^{2} b_{z}-u \frac{\partial B_{z}^{0}}{\partial x}-v \frac{\partial B_{z}^{0}}{\partial y}=\operatorname{Re}_{\omega} \frac{\partial B_{z}^{0}}{\partial t},
$$

where the induced magnetic field $b_{z}$ has been normalized by $\mathrm{Rm} B_{m}$. Once $b_{z}$ is determined, Ampère's law gives an expression to calculate electric currents, namely, $j_{x}=\partial b_{z} / \partial y$, $j_{y}=-\partial b_{z} / \partial x$. This equation also guarantees that the electric current density is divergence-free, $\nabla \cdot \mathbf{j}=0$.

The harmonic motion of the external magnet is described by the equation

$$
x(t)=D \sin (t)
$$

where $D=A / d$ is a geometrical parameter defined as the ratio of the amplitude of the oscillation, $A$, and the characteristic length scale.

We assume that the induced field is zero at a long enough, finite distance from the source of the applied field. Therefore, we impose the condition that the single component of the induced field vanishes at all boundaries. We look for numerical solutions using a formulation based on the primitive variables, the velocity and pressure, and the induced magnetic field as electromagnetic variable. The integration region is a square of $25 \times 25$ units (measured in terms of the characteristic length $d$ ). The magnetic obstacle oscillates around the geometrical center of the square in the $x$ direction. A finite difference method on an orthogonal equidistant grid of 636 $\times 636$ was used to solve the governing Eqs. (1)-(4), assuming a motionless fluid as initial condition and no-slip boundary conditions for the velocity components $(u=v=0)$ at all boundaries. The standard time-marching procedure described in [18] was extended to consider magntohydrodynamics (MHD) flows.

The analysis of the flow is based on the identification of critical points of the instantaneous streamlines that are generated in each cycle. In order to find the position and geometrical characteristics of the critical points as functions of time, we follow the methodology presented by $[16,19]$, which consists in determining the curvature fields of the instantaneous streamlines, and identifying the high curvature isolated points as critical hyperbolic or elliptic points of the flow. As explained in [16], near the critical points, the direction of fluid particle trajectories changes over short length scales. Therefore, it is expected that local maxima of curvature correspond to topologically special points of the flow. The nature of the critical points is found using the OkuboWeiss (OW) criterion in two dimensions [20]. In a region dominated by rotation (elliptical critical point), the enstrophy is larger than the squared strain rate and the OW parameter is positive. In contrast, if the local deformation dominates the flow in a small region, the OW parameter is negative indicating that the critical point is hyperbolic.

Two symmetries can be identified in the flow. First, given that the magnetic obstacle moves along the horizontal direction, and that the structure of the magnetic field is symmetric with respect to the same line, we have mirror symmetry with respect to the $y$ coordinate, i.e.,

$$
\begin{gathered}
u(x, y, \phi)=u(x,-y, \phi), \\
v(x, y, \phi)=-v(x,-y, \phi) .
\end{gathered}
$$

Second, after transients have died out, we have a cyclic symmetry in the flow such that the velocity field transforms according to the following reflection around the horizontal axis:

$$
\begin{gathered}
u(x, y, \phi) \rightarrow-u(-x, y, \phi-\pi), \\
v(x, y, \phi) \rightarrow v(-x, y, \phi-\pi) .
\end{gathered}
$$




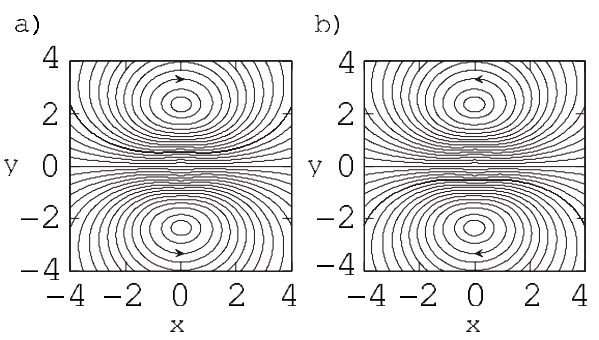

FIG. 1. Instantaneous streamlines for $D=0.01, \mathrm{Ha}=100$, and $\operatorname{Re}_{\omega}=1$ : (a) $\phi=-997 \pi / 1000$, (b) $\phi=3 \pi / 1000$. The magnetic obstacle is in the region $-0.5<x<0.5$ and $-0.5<y<0.5$.

\section{NUMERICAL RESULTS}

Since the most interesting flow dynamics is observed as the oscillation frequency changes, the range of oscillation Reynolds numbers explored is from 1 to 100 . In turn, the Hartmann number is fixed to a sufficiently high value ( $\mathrm{Ha}$ $=100$ ) so that MHD effects become relevant. Likewise, the dimensionless amplitude of oscillation of the localized magnetic field is fixed at $D=0.01$. The flow displayed a transient state that lasted 50 cycles. All results shown in this section correspond to long times after the onset of the motion, once the transient effects have died out. At long times, the motion is periodic and will be described as a function of the phase $(-\pi<\phi<\pi)$ in the cycle.

\section{A. Low oscillation frequencies $\left(\operatorname{Re}_{\omega}=1\right)$}

With $\operatorname{Re}_{\omega}=1$, the dominant pattern of the flow generated by the oscillatory localized magnetic field is a dipole-type flow composed of two counter rotating vortices located symmetrically above and below the average position of the imposed magnetic field. Dipole vortices can also be observed when a uniform flow passes through a nonoscillating magnetic obstacle, as discussed in $[7,8]$. These patterns are characteristic of flows generated by point momentum sources [21]. It is found that the direction of rotation of the dipole vortex is reversed twice per cycle, in such a way that each direction of rotation lasts half-cycle. The value $\phi=0$ is defined as the time where the magnet is at the point $(x=0, y=0)$.

In Fig. 1 we show instantaneous streamlines for flows with a relative phase of $\pi$, illustrating the symmetry described in Eq. (7). The topological changes leading to the reversal of the vortices are shown in Fig. 2. At $\phi=-927 \pi / 1000$ [Fig. 2(a)], the flow distribution is similar to that observed at $\phi=-997 \pi / 1000$ [see Fig. 1(a)], but with the instantaneous streamlines slightly perturbed near the average position of the magnet $(x=0, y=0)$. Then, at $\phi$ $=-895 \pi / 1000$ [Fig. 2(b)], a different picture is observed where four new critical points clustered near the origin appear. Two symmetric elliptic points are located vertically displaced above and below the origin; the direction of rotation of the fluid around these elliptic points is opposite to that of the vortical structures created in the previous half-cycle. Two hyperbolic points appear along the horizontal axis one at each side of the center of coordinates. The two hyperbolic critical points are linked by two heteroclinic invariant mani- a)

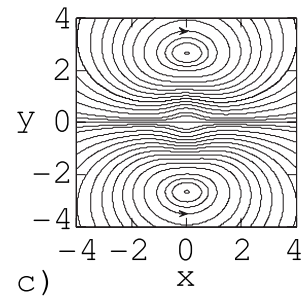

b)
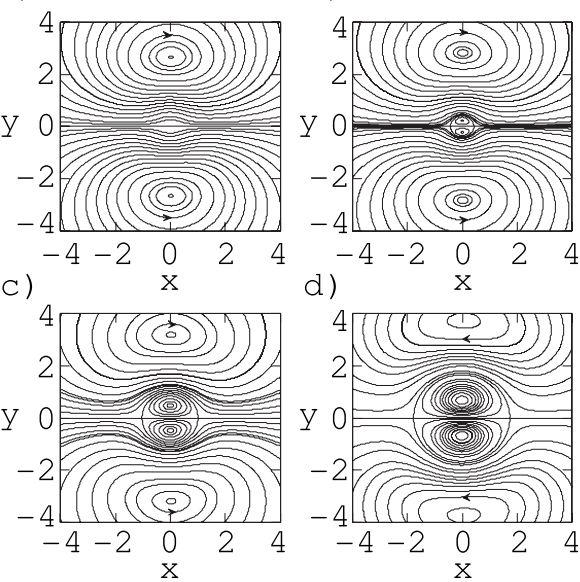

FIG. 2. Instantaneous streamlines for $D=0.01, \mathrm{Ha}=100$, and $\operatorname{Re}_{\omega}=1:$ (a) $\phi=-927 \pi / 1000$, (b) $\phi=-895 \pi / 1000$, (c) $\phi=-831 \pi / 1000$, and (d) $\phi=-752 \pi / 1000$.

folds that form a separatrix with isolated regions around the recently formed elliptical points. The topology of the flow pattern is similar at a later phase $(\phi=-831 \pi / 1000)$ as can be seen in Fig. 2(c), but the scale of the distribution of the critical points located around the center is enlarged. The original vortices generated in the previous cycle are pushed away from the center by the emerging structures and tend to dissipate due to viscous effects. The final stage of the flow evolution is displayed in Fig. 2(d) $(\phi=-752 \pi / 1000)$, where the hyperbolic points and the vortex pair formed in the previous cycle are almost out of the region shown in the figure and the vortex pair with opposite sense of rotation prevail. This cycle is repeated when the oscillating magnet changes its direction of motion; The thresholds where a new counter rotating vortex pair is created are $\phi_{0}=-896 \pi / 1000$ and $\phi_{0}=104 \pi / 1000$.

Figure 3 shows the evolution of the $x$ and $y$ positions of the critical points of the instantaneous streamlines as functions of time phase. We follow a pair of each elliptic and hyperbolic points that are cyclically generated, and move away from the average position of the magnet in the $y$ and $x$ directions, respectively. The initial and final velocities of the elliptical points are larger just after $\phi_{o}=-896 \pi / 1000$ and $104 \pi / 1000$. The velocity of the hyperbolic points is practically constant for the whole cycle.

The genesis of the critical points can be traced to the behavior of the axial velocity as a function of time $u(t)$ at the horizontal line $y=0$. At this line, the vertical velocity is zero due to the symmetry of the flow and the axial velocity evolves in time according to the profiles shown in Fig. 4. At $\phi=-376 \pi / 1000$ the velocity profile displays a single local maximum at approximately $x=0$. At subsequent times, the absolute value of the velocity becomes smaller and its distribution gradually develops a local minimum. This trend continues up to a time when the velocity distribution touches tangentially the $u=0$ line, indicating that a critical point appears close to the origin. At this time, the local maximum is the largest. At subsequent time instants, the velocity distribution crosses the $u=0$ line at two points (located nearly sym- 


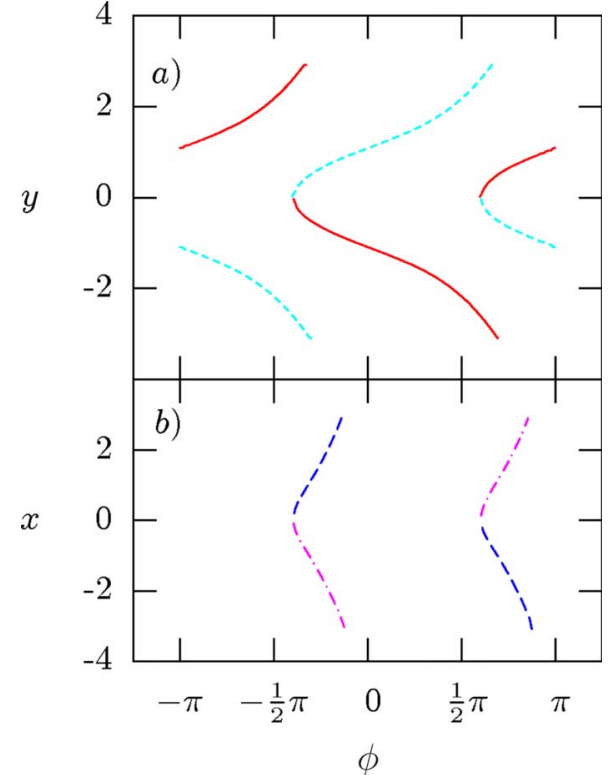

FIG. 3. (Color online) Position of critical points of the instantaneous streamlines for $D=0.01, \mathrm{Ha}=100$, and $\operatorname{Re}_{\omega}=1$ : (a) $y$ position of elliptical points as a function of phase $\phi$. Red (dark) and blue (light) lines refer to vortices rotating clockwise and counterclockwise, respectively. (b) $x$ position of hyperbolic points, as a function of the phase $\phi$.

metric around the origin) as can be appreciated in the inset of Fig. 4. These points correspond to the hyperbolic critical points shown in Figs. 2(b)-2(d). The small asymmetry displayed in the inset is generated by the fact that the flow moves in opposite directions for positive and negative phases, respectively.

\section{B. High oscillation frequencies $\left(\operatorname{Re}_{\omega}=100\right)$}

For $\operatorname{Re}_{\omega}=100$ and $\mathrm{Ha}=100$, the qualitative flow features in most of the cycle are similar to those described in the previous paragraphs for $\operatorname{Re}_{\omega}=1$. Specifically, the flow is dominated by two counter rotating vortices that switch directions of rotation twice per cycle. As in the previous example, the phase is referred to the motion of the magnet, with

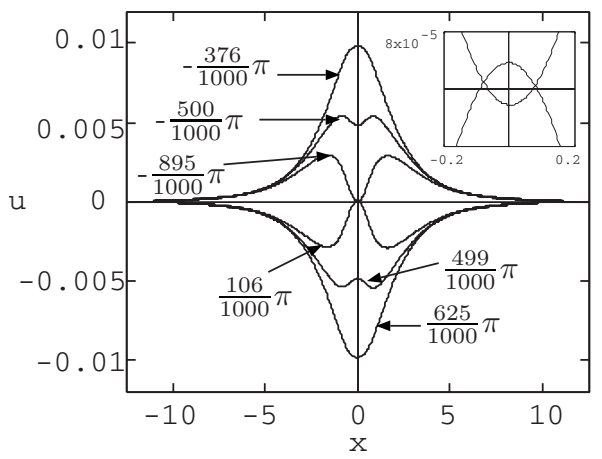

FIG. 4. $u$-velocity component as a function of the axial coordinate $x$ for $y=0$ for $\operatorname{Re}_{\omega}=1$. Labels near the lines indicate their phase in the cycle. The inset shows an amplification of the region close to the origin. a)

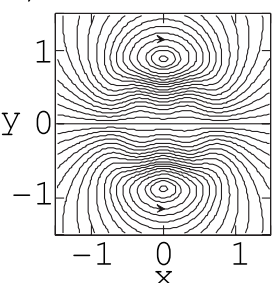

b)

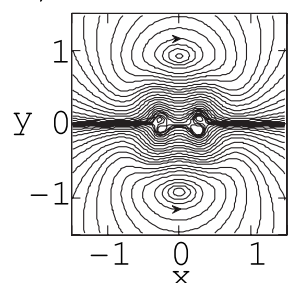

C)

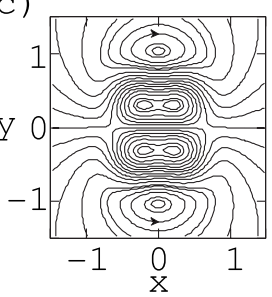

d)

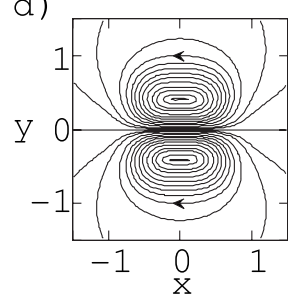

FIG. 5. Instantaneous streamlines for $D=0.01, \mathrm{Ha}=100$, and $\operatorname{Re}_{\omega}=100: \quad$ (a) $\phi=-553 \pi / 1000$, (b) $\phi=-542 \pi / 1000$, (c) $\phi=-534 \pi / 1000$, and (d) $\phi=-489 \pi / 1000$.

$\phi=0$ at the moment when the magnetic obstacle is at $(x=0, y=0)$ and has maximum velocity. However, for high $\mathrm{Re}_{\omega}$, the transformation is more complex and at a smaller scale as can be appreciated in Figs. 5-7. When the phase is close to $-553 \pi / 1000$, the speed of the flow is reduced at two points located on the horizontal axis and at the sides of the origin; eventually, two clusters of critical points with the same distributions as those found for $\operatorname{Re}_{\omega}=1$, i.e., four elliptical and four hyperbolic points, are formed inside the area covered by the magnetized zone. This distribution of critical points was not observed in the case $\operatorname{Re}_{\omega}=1$. The initial steps in the transition are illustrated in Figs. 5(a) and 5(b). At $\phi$ $=-542 \pi / 1000$ and for a short time interval smaller than $5 \pi / 1000$, the two sets of critical points are clearly separated with a small interval of the horizontal axis where the transversal velocity is very close to zero. This situation is illustrated in Fig. 5(b). As will be discussed in more detail below, in fact, the two recirculation zones do not appear simultaneously. A detailed analysis shows that the rightmost appears first, but only a very short time interval follows before the other is created. See the inset of Fig. 6 and the discussion of Fig. 7. Shortly after they are formed, the two clusters move

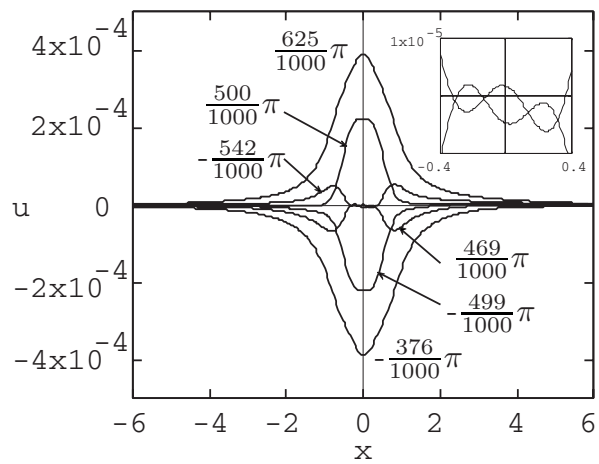

FIG. 6. $u$-velocity component as a function of the axial coordinate $x$ for $y=0$ for $\operatorname{Re}_{\omega}=100$. Labels near the lines indicate their phase in the cycle. The inset shows an amplification of the region close to the origin. 


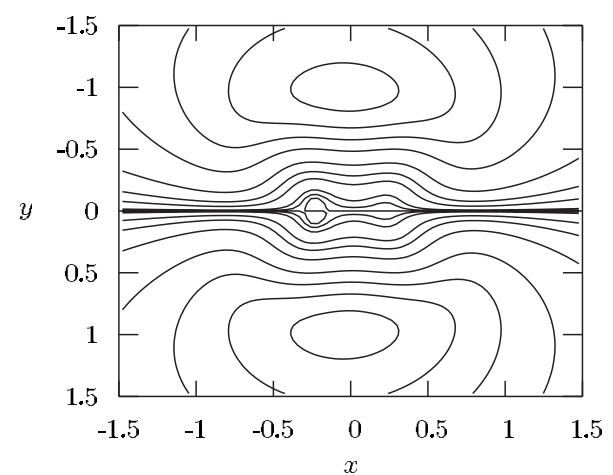

FIG. 7. Instantaneous streamlines for $D=0.01, \mathrm{Ha}=100$, and $\operatorname{Re}_{\omega}=100$ and $\phi=469 \pi / 1000$.

toward each other and merge to form structures of two elliptic points with a hyperbolic point in between, that move away from the $y=0$ line as illustrated in Fig. 5(c). The two hyperbolic points located further away from the origin in Fig. 5(b) move along the horizontal line. The last qualitative step in the evolution occurs when the two elliptic points and one hyperbolic point in each cluster combine to form a single elliptic point, suppressing the hyperbolic point. This configuration is topologically equivalent to that shown in Fig. 5(a), but the rotation direction is reversed. The instantaneous velocity profiles shown in Fig. 6 indicate the history of the formation of the critical points. Just as it was described in the case for smaller oscillatory Reynolds number, the asymmetry of the extrema of the curves that is more clearly shown in the inset of the figure is originated because the magnetic obstacle moves in opposite directions in each half-cycle. The fact that the flow is slightly asymmetric with respect to the vertical line $x=0$ indicates that there should be a phase interval (which might be very small), where there is only one cluster of two hyperbolic and two parabolic points located off the point $(x=0, y=0)$. This situation is neatly illustrated in Fig. 7 where the instantaneous streamlines that correspond to $\phi$ $=469 \pi / 1000$ are shown. According to the inset of Fig. 6 , at this phase, only one maximum has passed through the line $u=0$ and then only one set of two elliptic and two hyperbolic points, located at the left side of point $(x, y)=(0,0)$ are present.

\section{Bifurcation map}

The $\operatorname{Re}_{\omega}-\phi$ bifurcation map of qualitative behavior is shown in Fig. 8. The inset in the figure shows the region $-0.59 \pi<\phi<-0.51 \pi$ in greater detail. The symbols represent actual calculations and the lines separate regions with different qualitative flow patterns. The plane is divided into five regions. In the region above the line I there are no critical points in the immediate vicinity of the origin. In a larger region critical points are present in the flow, with those closest to the origin forming a vortex pair as in Fig. 1(a). As line I is crossed, a pair of counter rotating vortices are created. An example of the flow is given in Fig. 2(b). Line I passes through the point $\operatorname{Re}_{\omega}=1, \phi=-896 \pi / 1000$, which is consistent with Fig. 2. Above $\operatorname{Re}_{\omega}=50$, the flow transition is more complicated. The leftmost line in the inset is labeled with

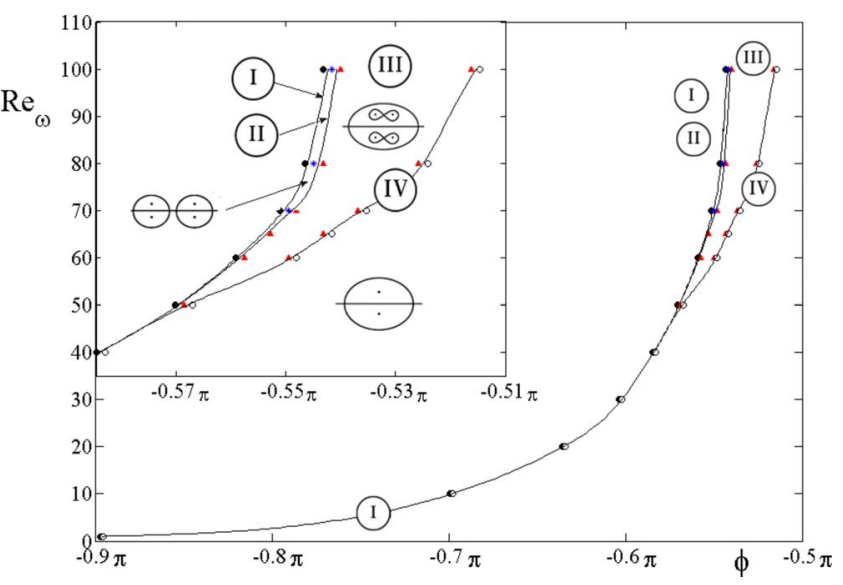

FIG. 8. (Color online) Bifurcation diagram for critical points of the instantaneous streamlines. Representative examples of flows are shown in Figs. 2 and 5.

symbols I and II, since the scale of the plot prevents us from resolving the two lines that separate an extremely narrow region where only one set of two pairs of two elliptic and two hyperbolic points are present. See Fig. 7. In the region between lines I and III, a pair of clusters of two elliptic and two hyperbolic points separated by a region of stagnant fluid are observed as illustrated in Fig. 5(b). The flow in the region between lines III and IV is characterized by the two pairs of two elliptic points separated by the homoclinic orbit of a hyperbolic point. Each of these structures are located above and below the horizontal line. An example of this flow is shown in Fig. 5(c). Finally, line IV separates regions with and without homoclinic orbits.

\section{THEORETICAL BIFURCATION ANALYSIS}

A local analysis of the streamline patterns near the symmetry line $y=0$ can be performed with the use of a normal form of the velocity field, i.e., a simplification of the field which nevertheless gives a qualitatively correct picture of the streamline pattern $[22,23]$.

For the present flow, the velocity field can be expanded in a Taylor series in $y$,

$$
\begin{gathered}
u=u_{0}(x)+u_{2}(x) y^{2}+u_{4}(x) y^{4}+\cdots, \\
v=-y\left(u_{0}^{\prime}(x)+\frac{1}{3} u_{2}^{\prime}(x) y^{2}+\frac{1}{5} u_{4}^{\prime}(x) y^{4}+\cdots\right),
\end{gathered}
$$

where

$$
u_{k}(x)=\frac{1}{k !} \frac{\partial^{k} u}{\partial x^{k}}(x, 0) .
$$

Only even-order terms of $y$ appear in $u$ due to the mirror symmetry of the velocity field in the line $y=0$. See Eq. (6). The expression for $v$ follows from $u$ using the equation of continuity.

The topology of the streamlines is in general given by the lowest-order terms of the velocity field. The lowest-order term in $u$ including both $x$ and $y$ is $u_{2}(x) y^{2}$, and assuming the 
nondegeneracy condition that this term does not vanish, we drop terms of higher order and replace $u_{2}(x)$ by a constant, which, after an appropriate scaling, can be chosen as 1 .

The line $y=0$ is a streamline and the velocity here is given by $u_{0}(x)$. We approximate this by a polynomial

$$
u_{0}(x)=c_{0}+c_{1} x+c_{2} x^{2}+\cdots+c_{n-2} x^{n-2}+\frac{1}{n} x^{n} .
$$

The truncation order $n$ is determined by the maximal number of stagnation points that can occur in the specific flow. Again, the value $1 / n$ is obtained by an appropriate scaling. In the present case, the relevant choice is $n=4$, as discussed in Sec. III, we observe up to four critical points on $y=0$. See also Figs. 5 and 6. The term of degree $n-1$ is omitted, since it can be removed by a translation of the origin.

Hence, we consider the normal form for the equations for the streamlines

$$
\begin{gathered}
\dot{x}=u=u_{0}(x)+y^{2}, \\
\dot{y}=v=-y u_{0}^{\prime}(x),
\end{gathered}
$$

with $u_{0}$ given by Eq. (10) and the $c_{k}$ being parameters. We note in passing that a rigorous derivation of this normal form can be obtained by perturbation methods following an approach previously used in similar situations [22-25].

For $n=4$ the bifurcation diagram is three-dimensional depending on the parameters $c_{0}, c_{1}, c_{2}$. Since the system Eqs. (11) is almost identical to the normal form for flow close to the axis in axisymmetric flow $[23,26]$, the bifurcation analysis here immediately follows from that case. Here we summarize the results. The parameter space is divided by surfaces into regions with different flow topologies. Two of these surfaces are

$$
R_{ \pm}: c_{1}^{2}=\left(\frac{2}{3}\right)^{3}\left(-c_{2} \pm \sqrt{\lambda}\right)\left(2 c_{2} \pm \sqrt{\lambda}\right)^{2}, \quad \lambda>0
$$

where

$$
\lambda=c_{2}^{2}+3 c_{0} .
$$

Intersecting $R_{+}$a recirculation zone is created, and at $R_{-}$two such zones merge into a single one. At

$$
S: c_{1}^{2}=-4\left(\frac{2}{3} c_{2}\right)^{3}, \quad \lambda<0
$$

a pair of critical points inside a recirculation zone are either created or destroyed.

The coefficients $c_{k}$ depend on the physical parameters which in the present flow are $\operatorname{Re}_{\omega}$ and $\phi$. Hence, the bifurcation diagram in the $\left(\operatorname{Re}_{\omega}, \phi\right)$ plane is found from a twodimensional slice in the $\left(c_{0}, c_{1}, c_{2}\right)$ parameter space, giving rise to bifurcation curves where the slice intersects the bifurcation surfaces $R_{ \pm}$and $S$. From the plots of $u_{0}(x)$, it appears that this function is very close to being symmetric. See Figs. 4 and 6. One would expect this symmetry to be more pronounced as the speed of the magnets tends to zero and eventually disappear for high magnet velocities. The function $u_{0}(x)$ is perfectly symmetric when $c_{1}=0$, and we hence expect that for the relevant slice $c_{1}\left(\operatorname{Re}_{\omega}, \phi\right)$ takes small values.

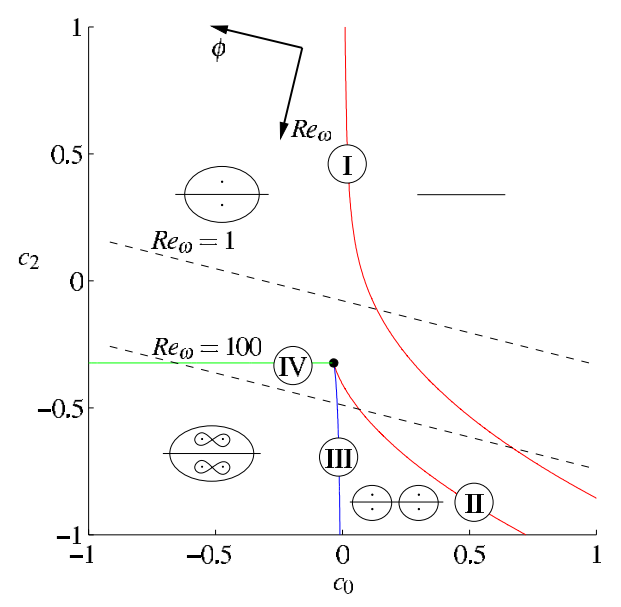

FIG. 9. (Color online) Theoretical bifurcation diagram in the $c_{2}, c_{0}$ parameter plane for $c_{1}=0.2$. The dashed lines correspond to the sequences of topologies in Figs. 2 and 5.

In Fig. 9 we show such a slice for a small constant value of $c_{1}$. The two curves I and II are intersections with the bifurcation surface $R_{+}$, the curve III is the intersection with $R_{-}$, and IV is the intersection with $S$. Three bifurcation curves emanate from a single point, denoted a codimension- 2 point. When $c_{1} \rightarrow 0$, the point moves toward I, and I and II coincide in the limit $c_{1}=0$.

The physical bifurcation diagram in the $\left(\operatorname{Re}_{w}, \phi\right)$ space is obtained from a smooth deformation of this diagram. The details will depend on the actual functional dependence between the physical and mathematical parameter, which cannot be inferred from the present analysis. However, from a simple linear relation between the two sets of parameter a bifurcation diagram which is qualitative consistent with the numerical results can be obtained. If the $\left(\operatorname{Re}_{\omega}, \phi\right)$ coordinate system is located as indicated in Fig. 9, the two series of simulations at $\operatorname{Re}_{\omega}=1,100$ intersect the bifurcation curves in agreement with Figs. 2 and 5. More detailed and quantitative information can be established from a rigorous perturbation approach [23]. The point we want to stress here is that the analysis shows that the set of streamline topologies obtained numerically is expected to be complete, and, furthermore, there is a natural partition of the vortex creation into two kinds: Either $\operatorname{Re}_{\omega}$ is below a certain threshold, and only a single pair of vortices occur or $\mathrm{Re}_{\omega}$ is above that threshold, and the vortex creation has an intermediate step with two vortex pairs. The threshold is obtained for the value of $\operatorname{Re}_{\omega}$ where the path passes through the codimension-2 point. From Fig. 8 this happens for $\mathrm{Re}_{\omega}$ slightly below 50 .

The different flow topologies are shown in Fig. 9.

\section{DISCUSSION AND CONCLUSIONS}

A numerical study of the two-dimensional flow generated by an oscillating magnetic obstacle reveals that in most of the oscillatory cycle, the flow is dominated by two counter rotating vortices with structure similar to the ones observed when a localized magnetic obstacle is in constant relative motion with respect to a thin layer of a conducting fluid like 
that described by $[6,8]$. However, when the magnetic obstacle oscillates, the vortex pair changes its direction of rotation twice per cycle undergoing a flow transformation involving the interplay of critical points in the instantaneous streamlines that results in the flow reversal. In each cycle, two new pair of vortices are created and those generated previously are pushed away from the time average of the position of the magnetic obstacle. As a new vortex pair is created twice during a cycle, from a strict mathematical point of view, there would eventually be an infinite array of vortices in the flow and they do not actually disappear as they move away from the axis but only dissipate to a very low strength. Given the specificities of the motion of the magnetic obstacle that we have chosen to study, the flow generated by the induced Lorentz force displays a cyclic symmetry and a spatial symmetry, which restricts the distribution of critical points in the flow. For larger oscillatory Reynolds numbers, the interplay of the sets of elliptic and hyperbolic critical points that appear near the time average position of the magnetic obstacle is increasingly complex as displayed in the map of qualitative behavior shown in Fig. 8. From the trend displayed in this figure, we speculate that for larger oscillator Reynolds numbers, the number of interspersed elliptic and hyperbolic points in each cluster located above and below the symmetry line should increase. The fact that the relatively simple local analysis described in Sec. IV gives a surprisingly complete picture of the qualitative features of the flow is due to the symmetries of the problem under analysis. Specifically, it is observed that the motion reduces to a one-dimensional flow along the line $y=0$. A key feature of the local analysis is that the expansion is truncated at fourth order. This is of course suggested by the fact that $u$-velocity profile at $y=0$ calculated numerically has four stagnation points. One must note however that the theoretical analysis is local and does not contain information on the outer vortical structures once they are expelled by the emerging vortices.

It is clear that a limitation of our model is the assumption that only the vertical component of the magnetic field has been considered. The horizontal components of the magnetic field give rise to three-dimensional effects; however, in a very shallow layer of electrically conducting fluid these components would be much smaller than the dominant vertical one. Therefore, to a good approximation the main characteristic behavior of the magnetohydrodynamic flow can be described by considering only the vertical component.

\section{ACKNOWLEDGMENTS}

S.C., E.R., and A.B. acknowledge support from CONACYT through Project No. 59977. A.B. also acknowledges support from CONACYT through a PhD grant.
[1] B. S. Williams, D. Marteau, and J. P. Gollub, Phys. Fluids 9, 2061 (1997)

[2] D. Rothstein, E. Henry, and J. P. Gollub, Nature (London) 401, 770 (1999).

[3] G. A. Voth, T. C. Saint, G. Dobler, and J. P. Gollub, Phys. Fluids 15, 2560 (2003).

[4] L. Rossi, J. C. Vassilicos, and Y. Hardalupas, J. Fluid Mech. 558, 207 (2006).

[5] L. Rossi, J. C. Vassilicos, and Y. Hardalupas, Phys. Rev. Lett. 97, 144501 (2006).

[6] Y. D. Afanasyev and V. N. Korabel, J. Fluid Mech. 553, 119 (2006).

[7] S. Cuevas, S. Smolentsev, and M. A. Abdou, J. Fluid Mech. 553, 227 (2006).

[8] S. Cuevas, S. Smolentsev, and M. A. Abdou, Phys. Rev. E 74, 056301 (2006).

[9] E. V. Votyakov, Yu. B. Kolesnikov, O. Andreev, E. Zienicke, and A. Thess, Phys. Rev. Lett. 98, 144504 (2007).

[10] E. V. Votyakov, E. Zienicke, and Yu. B. Kolesnikov, J. Fluid Mech. 610, 131 (2008).

[11] N. Riley, Annu. Rev. Fluid Mech. 33, 43 (2001).

[12] A. Beltrán, S. Cuevas, and E. Ramos, Magnetohydrodynamics 45, 173 (2009).
[13] R. Legendre, La Rech. Aéronaut. 54, 3 (1956).

[14] A. E. Perry and B. D. Fairlie, Adv. Geophys. 18, 299 (1974).

[15] S. Goto, D. R. Osborne, J. C. Vassilicos, and J. D. Haigh, Phys. Rev. E 71, 015301(R), 2005.

[16] N. T. Ouellette and J. P. Gollub, Phys. Rev. Lett. 99(19), 194502 (2007).

[17] M. McCaig, Permanent Magnets in Theory and Practice (Wiley, New York, 1977)

[18] M. Griebel, T. Dornseifer, and T. Neunhoeffer, Numerical Simulation in Fluid Dynamics (SIAM, Philadelphia, 1998).

[19] W. Braun, F. de Lillo, and B. Eckhardt, J. Turbul. 7, 1 (2006).

[20] J. Weiss, Physica D 48, 273 (1991).

[21] Y. D. Afanasyev and V. N. Korabel, Phys. Fluids 16, 3850 (2004).

[22] P. G. Bakker, Bifurcations in Flow Patterns (Kluwer, Dordrecht, 1991).

[23] M. Brøns, Adv. Appl. Mech. 41, 1 (2007).

[24] F. Gürcan and A. Deliceoğlu, Phys. Fluids 17, 093106 (2005).

[25] M. Brøns, B. Jakobsen, K. Niss, A. V. Bisgaard, and L. K. Voigt, J. Fluid Mech. 584, 23 (2007).

[26] M. Brøns, in Simulation and Identification of Organized Structures in Flows, edited by J. N. Sørensen, E. J. Hopfinger, and N. Aubry (Kluwer, Dortrecht, 1999), pp. 213-222. 